\title{
Phase Analysis for 2-D weakly coupled oscillatory systems
}

\author{
Alejandro Rojas and Richard Middleton
}

\begin{abstract}
A phase representation of a two dimensional nonlinear autonomous oscillator is presented. The validity of this representation is argued from the point of view of continuity and a global model is then obtained for a set of coupled oscillators. A brief review of a biological motivated 2 dimensional model for Calcium oscillations within a cell is presented as an example of this concepts. A discussion on the results obtained concludes the present work.
\end{abstract}

\section{INTRODUCTION}

The presence of coupled oscillatory activity is a wide spread phenomenon that can be found, to name just two examples, in biological processes and in power systems. In some cases oscillation is a desired behaviour, in others it is something that should be avoided, nonetheless it is frequently important to understand oscillatory dynamics. The behaviour of coupled oscillators is of particular interest in examples such as populations of Dictyostelium discoideum, living cells that display a coordinate behaviour through synchronism in order to ensure the continuity of their life cycle [14], or the beating of the human heart. In this latter example a lack of synchronism from the muscle cells can trigger fibrillation and cause death [13]. A third example is the load balance between power generators within an electric power grid, at the core of the United States East Coast huge blackout during 2003, [1].

Because of the common dynamics behind these examples many researchers have made fundamental contributions to the study of coupled oscillators. [11], [12], [2] or [8] consider the case of a family of oscillators governed by a phase model defined as:

$$
\dot{\phi}_{i}=\omega_{i}+\frac{K}{N} \sum_{j=1}^{N} \sin \left(\phi_{j}-\phi_{i}\right)
$$

where $\phi_{i}$ is the natural frequency of the $i$-oscillator, $K$ is the coupling strength and $N$ the number of oscillators involved. Note that the Kuramoto model, see [11] (as equation (1) is often called), by definition, assumes a global coupling in which the dynamics of each oscillator are affected equally by all the other oscillators. This is a quite strong assumption and in many cases we will face specific geometric dependencies in the coupling, also know as lattices, that are at odds with the globality assumed in the Kuramoto model.

The analysis in this paper explores this issue focusing on 2D oscillatory systems with a couple of features: (a) 'Strongly attractive' stable limit cycles and (b) 'Weak' non-global couplings. The Poincaré map is used later in sections II and III to define these features. A similar exercise, but with different mathematical tools can also be found in [9].

Section II present the phase model given a state space description and the authors definition for "attractiveness". Section III includes the perturbation effect of a coupling function (also stated in terms of the state space variables) plus a useful definition for

This work was supported by The Centre for Complex Dynamic Systems and Control,The University of Newcastle , 2308, Australia

A. Rojas is with the School of Electrical Engineering and Computer Science, The University of Newcastle, 2308, Australia ajrojasnestudentmail.newcastle.edu.au

R. Middleton is with the School of Electrical Engineering and Computer Science, The University of Newcastle, 2308, Australia rick@ee.newcastle.edu.au "weak coupling" based on the concept of "attractiveness. Section IV presents the main result of this paper, by which a continuum of oscillator can be described by a global dynamic. Section V presents, as an illustrative example, the model developed in [5] for cellular Calcium oscillations. Section VI concludes this work and list future developments on the present ideas.

\section{PhASE REPRESENTATION AND DISCRETE DESCRIPTION.}

Consider the following two dimensional autonomous oscillators:

$$
\dot{v}=f(v, \mu)
$$

Where $v \in \Re^{2}$ and $\mu \in \Re$ is the bifurcation parameter. Without loss of generality it is therefore assumed that each oscillator undergoes a supercritical Hopf bifurcations at $\mu=\mu_{1}$ and $\mu=\mu_{2}$. Outside this region there are no oscillations in the system. For a complete review on nonlinear oscillations, dynamical systems and bifurcation concepts the reader is referred to [6] or [7]. For this type of oscillators the presence of a stable limit cycle is at the base of the phase representation. If the limit cycle translated to contain the origin, then the limit cycle itself can be considered as a rotating vector. In a polar coordinate description, the angular component may be taken as a representation of the phase of the oscillator. To realize the translation itself, a useful point to consider is the equilibrium point $v_{e q}$, obtained from solving equation (2) when its RHS is set to zero. If the equilibrium is non-unique this procedure can be difficult, for this reason the discussion is limited to a two dimensional oscillator with only one equilibrium point. In this case this point will always lay inside the limit cycle and the shifting will be readily achieved subtracting it from $v(t)$. The equivalent phase description on the limit cycle would become:

$$
\dot{\phi}=g(\phi(t), \mu)
$$

This continuous representation in equation (3) will not be able to represent the whole behaviour described by (2). It will only be equivalent to the original model for trajectories close to the stable limit cycle subjected to small perturbations. Let introduce the following definition for the limit cycle (from [10]):

$$
\gamma=\left\{v \in \Re^{2} \mid v=u(t), 0 \leq t \leq T\right\}
$$

In which the periodic orbit $\gamma$ is the image of $u(t)$ in the state space. A suitable measure of distance from the limit cycle becomes then:

$$
\operatorname{dist}(v, \gamma)=\inf _{y \in \gamma}\|v-y\|
$$

And a neighbourhood, $U_{\xi}$, of the limit cycle will be a region of the state space defined as:

$$
U_{\xi}=\left\{v \in \Re^{2} \mid \operatorname{dist}(v, \gamma)<\xi\right\}
$$

Equation (3) is equivalent to (2) for all trajectories that, under the effect of small perturbations, remain inside $U_{\xi}$.

We assume, then, from the stability of the limit cycle that we can decompose the time varying linear system that describes this dynamics about $\gamma$ into a portion along the limit cycle, and a part orthogonal to the limit cycle. Furthermore, we also assume that the orthogonal portions are an exponentially stable time varying 
system attractive to the origin, with time scale much faster than that of the oscillations themselves.

In order to formalise a condition for such attractiveness, we will make use of the Poincaré map, see [10] for more details. This is a useful tool to tests stability of limit cycles. It does so by means of mapping after a cycle the points of a section transversal to $\gamma$ (i.e any line through $y \in \gamma$ that is not tangent to $\gamma$ at $y$ ), close to $\gamma$. Given a point $y_{o} \in \gamma$, the mapping of a starting point $p$ in such subset can be defined in terms of a scalar $\lambda$ mapping its position on the transversal section after a cycle in terms of its present position:

$$
p\left(t_{o}+T\right)=\lambda\left(t_{o}\right) \cdot p\left(t_{o}\right)
$$

For $p\left(t_{o}\right)$ in the vicinity of the limit cycle, $T$ period of time for the trajectory that started at $p$ to hit the transversal section after a cycle and $t_{o}$ an arbitrary time (that is implicitly defined by $y_{o} \in \gamma$, the chosen point over the limit cycle for which the Poincaré Map is obtained). The value of $\lambda$ will be different for different positions over the limit cycle, but nonetheless a proper study over a complete period will give us a complete picture of it as a periodic time function.

The following definition of attractiveness make use of the results from the Poincaré map:

Definition 1: (Attractiveness) Given the Poincaré map for the limit cycle under study and choosing the transversal region to be perpendicular at each point $y_{o}$, (i.e. given a $\lambda\left(t_{o}\right)$ for the $i$ oscillator), attractiveness is defined as:

$$
\lambda=\max \left\{\lambda\left(t_{o}\right)\right\}, \quad t_{o} \in\left[0, T_{i}\right]
$$

Strong attractiveness is obtained whenever $\lambda \ll 1$.

For a more complete exposition on the topic of stability for periodic orbits the reader is referred to [10].

For computational reasons we use an Euler method to obtain an approximation of (3). This is important for developments in section (3) and will prove to be quite accurate in the presence of the suggested condition of attractiveness and small perturbations.

$$
\begin{aligned}
\frac{\phi(k h+h)-\phi(k h)}{h} & =g(\phi(k h), \mu) \\
\phi(k h+h) & =\phi(k h)+\underbrace{h g(\phi(k h), \mu)}_{\tilde{g}(\phi(k h), \mu)} \\
\phi[k+1] & =\phi[k]+\tilde{g}(\phi[k], \mu)
\end{aligned}
$$

If we arbitrarily define an initial discrete time as the constant value $k_{o}$, then the phase value at $k+1$ can be written as:

$$
\phi[k+1]=\phi\left[k_{o}\right]+\sum_{j=0}^{k-k_{o}} \tilde{g}(\phi[k-j], \mu)
$$

\section{COUPLING AS A PHASE PERTURBATION.}

Suppose we take the dynamical model presented in equations (2) for an individual oscillator and in the case of a linear ring of oscillators coupled together with their nearest neighbours only. Label the oscillators in sequence, one of them with subindex $i$ and likewise the two neighbours with sub-indexes $i+1$ and $i-1$, with $i=1, \cdots N$ and $v_{-1} \triangleq v_{N}, v_{N+1} \triangleq v_{1}$. The coupling under study, without loss of generality, will appear as an extra term added to the first state variable, as:

$$
\dot{v}_{i}^{1}=f_{i}\left(v_{i}, \mu_{i}\right)+I_{i}(t)
$$

$I_{i}(\cdot)$ is the coupling function for the $i$ th-oscillator. If this coupling term is set to zero then the phase behaviour falls back to $N$ independent oscillators of the form (10). On the other hand, if this term is not zero it will introduce a variation in the phase trajectory in what can be exploited to perform a perturbation analysis, see also on this topic for example [3], [4]. This situation can be better described by assuming a function $\tilde{g}_{H}$, much like $\tilde{g}$, but also a function of $H$, where $H$ is the strength of the coupling $I_{i}(t)$, for each time interval $h$ (therefore becoming a quantised version of the continuous time coupling function).

$$
\begin{aligned}
\phi_{i}[k+1] & =\phi_{i}[k]+\tilde{g}_{H_{i}}\left(\phi_{i}[k], \mu_{i}, I_{i}[k]\right) \\
& =\phi_{i}[k]+\tilde{g}_{i}\left(\phi_{i}[k], \mu_{i}\right)+ \\
& +\Delta \tilde{g}_{i}\left(\phi_{i}[k], \mu_{i}, I_{i}[k]\right)
\end{aligned}
$$

where:

$$
\begin{aligned}
& \Delta \tilde{g}_{i}\left(\phi_{i}[k], \mu_{i}, I_{i}[k]\right)= \\
& \tilde{g}_{H_{i}}\left(\phi_{i}[k], \mu_{i}, I_{i}[k]\right)-\tilde{g}_{i}\left(\phi_{i}[k], \mu_{i}\right)
\end{aligned}
$$

Note 1: by definition $\tilde{g}_{H_{i}}=\tilde{g}_{i}$, whenever $H=0$ (no coupling present), or, in a similar way, $\Delta \tilde{g}=0$ if $I_{i}(\cdot)=0$, i.e. an height of zero for the coupling function.

Again (13) can be iterated to obtain:

$$
\begin{aligned}
& \phi_{i}[k+1]=\phi_{i}\left[k_{o}\right]+ \\
& \quad+\sum_{j=0}^{k-k_{o}} \tilde{g}_{H_{i}}\left(\phi_{i}[k-j], \mu_{i}, I_{i}[k]\right) \\
& \quad=\phi_{i}\left[k_{o}\right]+\sum_{j=0}^{k-k_{O}} \tilde{g}_{i}\left(\phi_{i}[k-j], \mu_{i}\right)+ \\
& \quad+\sum_{j=0}^{k-k_{o}} \Delta \tilde{g}_{i}\left(\phi_{i}[k-j], \mu_{i}, I_{i}[k]\right)
\end{aligned}
$$

This description was inspired by the work from [8], in which a more broad discussion of pulse-coupled biological inspired oscillators is presented. The potential of such a description is to isolate the effect of the coupling, described in equation (13), considering it directly as an added term in equation (10). Finally, for this section, a definition to quantify the "weakness" of the coupling strength is required in order to maintain valid the equivalence between models (2) and (3) under the perturbation effect introduced by the coupling. Weak coupling is therefore defined as:

Definition 2: (Weak Coupling) given a function $I(t)$ as the coupling for the $i$-oscillator. Consider it as a collection of pulses with variable strength $H$, as suggested in equation (12). Define also, $U_{\xi_{i}}$ as the neighbourhood of $\gamma_{i}$, the stable limit cycle for the $i$ thoscillator. Given $y_{o} \in \gamma_{i}$ the point in which a coupling of strength $H$ is applied and $(\cdot)_{\perp}$ the projection over the perpendicular (to the limit cycle) section at point $y_{o}$, weak coupling will be achieved if the trajectory perturbed in an amount $H$ is still inside $U_{\xi_{i}}$ after a cycle.

$$
\begin{aligned}
& \lambda_{i} \cdot \operatorname{dist}\left(y_{o}+(H, 0), y_{O}\right)_{\perp}<\xi_{i} \\
= & \lambda_{i} \cdot\left(\sqrt{\left(y_{O}^{1}+H-y_{O}^{1}\right)^{2}+\left(y_{O}^{2}-y_{O}^{2}\right)^{2}}\right)_{\perp}<\xi_{i} \\
= & \lambda_{i} \cdot|H|_{\perp}<\xi_{i}
\end{aligned}
$$

Where $\lambda_{i}$ is the scalar that quantifies the attractiveness of $\gamma_{i}$. The worst case scenario would be the maximum possible value for $|H|_{\perp}$. The obtained definition is then restated as:

$$
\lambda_{i} \cdot \max \left\{|H|_{\perp}\right\}<\xi_{i}
$$

Weak coupling will be obtained then, only if the maximum perpendicular component (with respect to the limit cycle) of the perturbed trajectory due to the coupling does not exceed the capacity of a given system to attract the same back onto the limit cycle in less than a period.

The main effect on an oscillator with properties of attractiveness and weak coupling is that the uncoupled features of $\gamma$ and equilibrium point location will still be valid even after the coupling takes place. For a more complete view on the concepts involved in 
the definition of attractiveness and weak coupling, as for example the Poincare map, the reader is referred to [10].

\section{Global Model}

In order to deduce an explicit model for the phase dynamic that lies behind (2) the nonlinear transformation $C$ suggested in section (2) is applied, switching the original description to a polar coordinates description.

$$
\begin{aligned}
& r_{i}=C_{r}\left(v_{i}\right)=\sqrt{\left(v_{i}^{1}\right)^{2}+\left(v_{i}^{2}\right)^{2}} \\
& \phi_{i}=C_{\phi}\left(v_{i}\right)=\operatorname{atan} 2\left(v_{i}^{1}, v_{i}^{2}\right)
\end{aligned}
$$

where $v_{i}=\left(v_{i}^{1}, v_{i}^{2}\right) \in \Re^{2}$ and $\operatorname{atan} 2(\cdot, \cdot)$ is the four quadrant arc-tangent.

Since we are only interested in the phase component, for any generic system like in (2) we will have a net reduction in dimensionality of the problem (from a set of two ODEs, one for each component of $v_{i}$, to a single expression for $\phi_{i}(t)$ ). However when we consider coupling, we can claim this reduction in dimensionality if and only if the coupling term can be satisfactorily describe by the phases of the oscillators alone that is, for example:

$$
I_{i}(t) \stackrel{C}{\Rightarrow} I\left(\phi_{i+1}(t), \phi_{i}(t), \phi_{i-1}(t)\right)
$$

If, on the other hand, the resulting dependency for $I$, due to its original definition in the state space, is both on the radius and the phase, then after the transformation we will have that for example:

$$
I_{i}(t) \stackrel{C}{\Rightarrow} I\left(\left(r_{i+1}(t), \phi_{i+1}(t)\right),\left(r_{i}(t), \phi_{i}(t)\right),\left(r_{i-1}(t), \phi_{i-1}(t)\right)\right)
$$

This does not preclude the synthesis of a model for $\phi_{i}$, but in the presence of coupling, it will require also the simultaneous synthesis of a model for $r_{i}$, in order to compute at each instant of time the example term described in (19). It follows then that if the coupling term $I$ cannot be alternatively described only by the phase of the involved oscillators, then we will not always experience a reduction in the dimensionality of the problem, on the other hand, we will still be able to obtain expressions for each oscillator phases. In an approach that involves $N$ countable oscillators, we will have that $v_{-1} \triangleq v_{N}, v_{N+1} \triangleq v_{1}$, as seen in section III. On the other hand, since each oscillator can be now located in space, the original index $i$ can be reinterpreted as $x_{i}$, position in space, with $x_{1}=0 \equiv i=1$ and $x_{N}=1 \equiv i=N$, resulting in a ring of length one. Under the assumption that the oscillators are evenly distributed on a ring, the information about the actual length of the ring is implicitly contained in the domain range for the natural frequencies distribution $\omega\left(x_{i}\right)$. Without loss of generality, we can always scale the linear dimensions so that the domain of $x_{i}$ is $[0,1]$.

Theorem 1: Given a population of $N$ evenly distributed identical weakly coupled oscillators with strongly attractive limit cycles, as in equation (12). Given also a general coupling term $I\left(t, x_{i}\right)$ for the $i$-oscillator (with $v_{0}=v_{N}$ and $v_{N+1}=v_{1}$ ). The resulting average phase behaviour, when the population number $N$ tends to infinity, will be determined by the following global dynamic:

$$
\begin{aligned}
\dot{\bar{\phi}}(t)=\int_{0}^{1} g & (\phi(t, x), \omega(x)) d x+ \\
& +\int_{0}^{1} \Delta g(\phi(t, x), \omega(x), I(t, x)) d x
\end{aligned}
$$

Where $\bar{\phi}(t)$ is:

$$
\bar{\phi}(t)=\int_{0}^{1} \phi(t, x) d x
$$

$\omega(x)$ is the continuous space distribution of natural frequencies for the uncoupled oscillators, and $I(t, x)$ is the limit:

$$
I(t, x)=\lim _{N \rightarrow \infty} I\left(t, x_{i}\right)
$$

The continuous space distribution for the coupling at each instant $t$.

Proof : Consider a description such as (12) for an oscillator at location $x_{i}$ :

$$
\begin{aligned}
\phi\left[k+1, x_{i}\right] & =\phi\left[k, x_{i}\right]+\tilde{g}\left(\phi\left[k, x_{i}\right], \mu\left[x_{i}\right]\right)+ \\
& +\Delta \tilde{g}\left(\phi\left[k, x_{i}\right], \mu\left[x_{i}\right], I\left[k, x_{i}\right]\right)
\end{aligned}
$$

Define the average:

$$
\bar{\phi}_{N}[k]=\frac{1}{N} \sum_{i=1}^{N} \phi\left[k, x_{i}\right]
$$

We will have:

$$
\begin{aligned}
\bar{\phi}_{N}[k+1] & =\bar{\phi}_{N}[k]+\frac{1}{N} \sum_{i=1}^{N} \tilde{g}\left(\phi\left[k, x_{i}\right], \mu\left[x_{i}\right]\right)+ \\
& +\frac{1}{N} \sum_{i=1}^{N} \Delta \tilde{g}\left(\phi\left[k, x_{i}\right], \mu\left[x_{i}\right], I\left[k, x_{i}\right]\right)
\end{aligned}
$$

The frequency of the oscillator is determined by the bifurcation parameter, therefore a proper map exists and it is invertible.

$$
s\left(\mu\left[x_{i}\right]\right)=\frac{\omega\left[x_{i}\right]}{2 \pi}, \quad \mu\left[x_{i}\right]=s^{-1}\left(\frac{\omega\left[x_{i}\right]}{2 \pi}\right)
$$

This replaced in the main expression permits to change the dependency on $\mu\left[x_{i}\right]$ for a dependency on $\omega\left[x_{i}\right]$ (switching from an implicit dependency on frequency to an explicit dependency on frequency). Taking now $\Delta x=1 / N$ and letting the number of oscillators $N$ tends to infinity, an integral definition come in place as the limit of a Riemann sum:

$$
\begin{aligned}
\bar{\phi}[k] & \hat{=} \lim _{N \rightarrow \infty} \bar{\phi}_{N}[k] \\
& =\lim _{N \rightarrow \infty} \frac{1}{N} \sum_{i=1}^{N} \phi\left[k, x_{i}\right] \\
& \equiv \lim _{\Delta x \rightarrow 0} \sum_{i=1}^{1 / \Delta x} \phi\left[k, x_{i}\right] \Delta x=\int_{0}^{1} \phi[k, x] d x
\end{aligned}
$$

Therefore:

$$
\begin{aligned}
\bar{\phi}[k+1] & =\bar{\phi}[k]+\int_{0}^{1} \tilde{g}(\phi[k, x], \omega(x)) d x+ \\
& +\int_{0}^{1} \Delta \tilde{g}(\phi[k, x], \omega(x), I[k, x]) d x
\end{aligned}
$$

If we divide the obtained expression for $\bar{\phi}[k+1]$ by $h$ (the sampling time), $h \in \Re^{+}, h \neq 0$, we have:

$$
\begin{array}{r}
\frac{\bar{\phi}(k h+h)-\bar{\phi}(k h)}{h}=\frac{1}{h} \int_{0}^{1} \tilde{g}(\phi(k h, x), \omega(x)) d x+ \\
+\frac{1}{h} \int_{0}^{1} \Delta \tilde{g}(\phi(k h, x), \omega(x), I(k h, x)) d x
\end{array}
$$

Recalling, from equation (9), the approximation involved in the term $\tilde{g}$ :

$$
\tilde{g}(\phi(k h, x), \omega(x))=h \cdot g(\phi(k h, x), \omega(x))
$$

And claiming for $\Delta \tilde{g}$, by a similar reasoning to that in (9), that:

$$
\Delta \tilde{g}(\phi(k h, x), \omega(x))=h \cdot \Delta g(\phi(k h, x), \omega(x))
$$

We obtain the following expression:

$$
\begin{aligned}
& \frac{\bar{\phi}(k h+h)-\bar{\phi}(k h)}{h}=\int_{0}^{1} g(\phi(k h, x), \omega(x)) d x+ \\
& \quad+\int_{0}^{1} \Delta g(\phi(k h, x), \omega(x), I(k h, x)) d x
\end{aligned}
$$

Finally, taking the limit when $h \rightarrow 0$ on this last expression will give us the result of the theorem. 


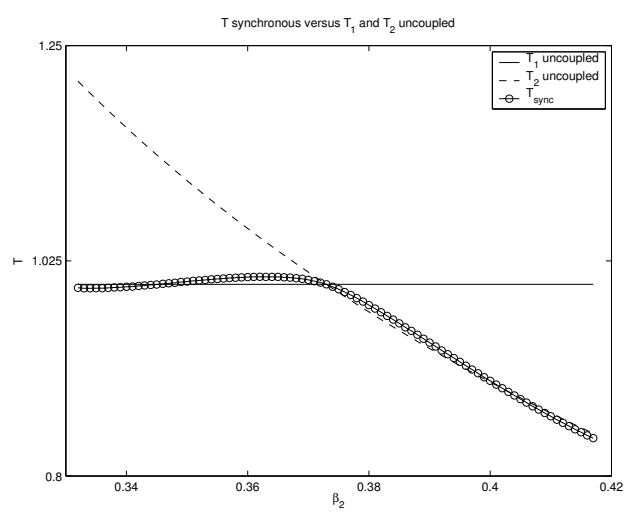

Fig. 1. $T_{\text {sync }}$ versus $T_{1}$ and $T_{2}$.

\section{EXAMPLE.}

We begin by reviewing a state space model presented in [5] for Calcium concentration oscillations within a cell. The cytosolic Calcium is labelled as the variable $Z$, similarly, $Y$ corresponds to the concentration of Calcium inside the special store within the cell (sometimes also called "pool"). The system of nonlinear ordinary differential equations (nonlinear ODEs) that describes the relationship between this two variables is presented next:

$$
\begin{aligned}
& \dot{Z}=V_{i n}-V_{a}+V_{b}+k_{f} Y-k Z \\
& \dot{Y}=V_{a}-V_{b}-k_{f} Y
\end{aligned}
$$

Where

$$
\begin{aligned}
V_{i n} & =\nu_{o}+\nu_{1} \beta \\
V_{a} & =V_{M 2} \frac{Z^{n}}{\left(K_{2}^{n}+Z^{n}\right)} \\
V_{b} & =V_{M 3} \beta \frac{Z^{w}}{\left(K_{a}^{w}+Z^{w}\right)} \frac{Y^{m}}{\left(K_{r}^{m}+Y^{m}\right)}
\end{aligned}
$$

Unless otherwise stated, the values for the different parameters in (20) and (21) are kept constant throughout the analysis, with exception of $\beta$ (the degree of saturation of the inositol $1,4,5$ trisphosphate, $I P_{3}$, receptor located on the surface of the cell. These values are presented in the appendix section.

Numerically, it can be shown that the system exhibits a stable limit cycle whenever the parameter $\beta$ is increased from 0.2368 $\left[\mu \mathrm{Mmin}^{-1}\right]$, until a second critical value of $0.7743\left[\mu \mathrm{Mmin}^{-1}\right]$ is reached, at which point the stable limit cycle vanishes into a stable equilibrium.

The case of a pair of cells, under the same settings for all constant parameters with exception of their $\beta$ values is here studied. First of all one of them is labelled as oscillator 1 and its parameter $\beta_{1}$ is fixed at $0.373\left[\mu \mathrm{Mmin}^{-1}\right]$. Meanwhile the other one, similarly denoted oscillator 2 , is allowed to vary its value of $\beta$ (that is a variable $\beta_{2}$ within a range in which phaselock is maintained). The coupling function for the case of a pair of oscillators is a reduced version of the more general definition presented in (11):

$$
I=I_{1}=Z_{2}-Z_{1}=-I_{2},
$$

Where $I_{1}$ is the coupling function for the first oscillator and $I_{2}$ is the coupling function has seen by the second oscillator. In general by the term coupling function $I_{z}$ we will be referring to the coupling function defined for the first oscillator. The set of values for $\beta_{2}$ in which the coupled system shows a common period for all its signals range between $\left[0.332\left[\mu \mathrm{Mmin}^{-1}\right], 0.417\left[\mu \mathrm{Mmin}^{-1}\right]\right]$.
In this range the period of the coupled system itself ( $\left.T_{\text {sync }}\right)$ does not necessarily correspond to the period of either of the two individual oscillators. Nonetheless simulations for this case show

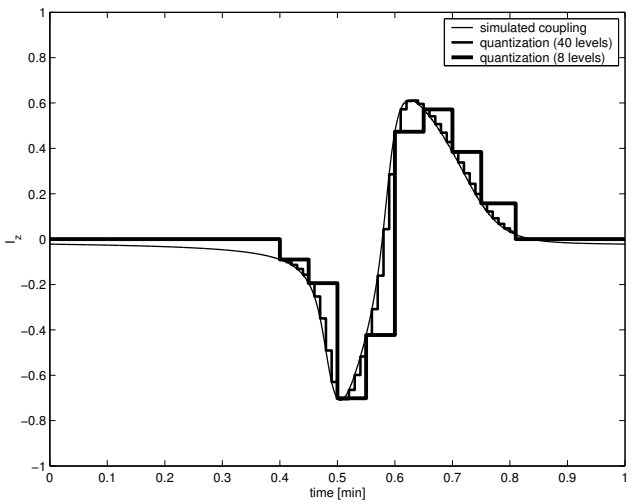

Fig. 2. $I_{z}$ and its quantised approximations.

that roughly $T_{\text {sync }}$ is close to the period of the first uncoupled cell $\left(T_{1}\right)$ for $\beta_{2}<0.373$, and vice-versa it is close to the period of the second uncoupled cell, for $\beta_{2} \geq 0.373$. To reduce the effect that $I_{z}$ has on the overall period a proper selection of $\beta_{2}=0.35\left[\mu \mathrm{Mmin}^{-1}\right]$ is performed guaranteeing a value of $T_{\text {sync }}=1[\mathrm{~min}]$. This achieves the double objective of testing the accuracy of phase prediction in a more ideal setting (centred only on the phase repercussions of $I$ and discarding its period change influence) and to keep the resulting visualisation simple, since the period will correspond to one minute. Under this conditions the coupled system is simulated and the time evolution of the coupling function $I_{z}$ is observed (one period presented in Figure 2). It can be seen that most of the signal is located in a time window of 0.4 [min] length (starting from 0.4 [min] until 0.8 [min]). A first simplification calls to neglect its value outside this window, and since we know that the effects of $I_{z}(t)$ are related with its height a quantisation is also required. Figure 2 shows the cases of eight levels and forty levels superimposed with the simulated coupling signal. The following phase prediction is going to be based on the eight level quantisation, since the 40 level would only demand more calculations but would not add to the discussion. Considering the selection of the eight level quantisation, the proper

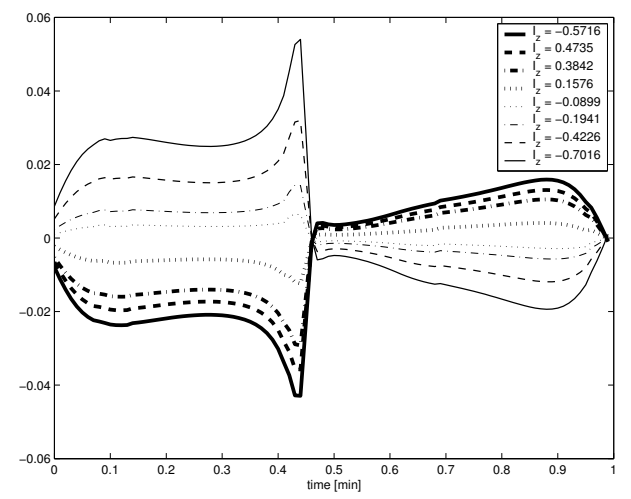

Fig. 3. First cell $\Delta g$ term for each level of the eight levels approximation of $I_{z}$

$\Delta \tilde{g}\left(\phi_{1}[k], \omega(1), I_{1}[k]\right)$ function will be a composed version of 
its continuous counterpart $\Delta g\left(\phi_{1}(t), \omega(1), I_{1}(t)\right)$, much like equation (9) is to equation (3). To obtain prior knowledge on the response of the first cell, the first oscillator has been tested, for each of the eight possible heights of $I_{z}$, with pulses in $I_{z}$ of width $h=0.01$ [min]. In Figure (3) the results is presented for all the levels.

Next, the phase of the uncoupled oscillator one has been subjected to three of the quantised version of $I_{z}$ located at absolute times 6 [min], 7 [min] and 8 [min] respectively. The effect can be appreciated in Figure 4 in which solid line is the simulated result under the coupling perturbation and the circled line is the predicted behaviour using the $\Delta \tilde{g}\left(\phi_{1}[k], \omega(1), I_{1}[k]\right)$ description. To test the accuracy of the prediction presented in Figure 4 a suitable error definition can be introduced:

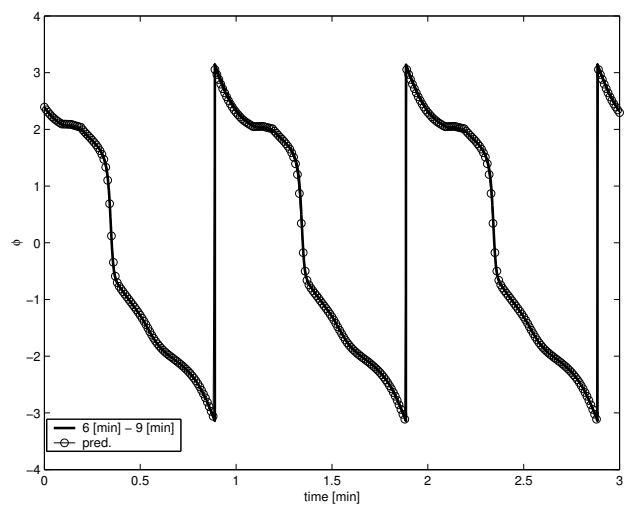

Fig. 4. Simulated (solid line) and predicted (circled line) phase response under coupling effect.

$$
e[k]=\frac{\left|\phi_{1}[k]-\hat{\phi}_{1}[k]\right|}{2 \pi} * 100
$$

where $\phi_{1}[k]$ is the simulated behaviour, $\hat{\phi}_{1}[k]$ is the predicted phase and the $2 \pi$ factor weight the error against the modulo $2 \pi$ condition of the phase. The result obtained is presented in Figure 5. The resulting peaks in error are mostly located in the steepest part

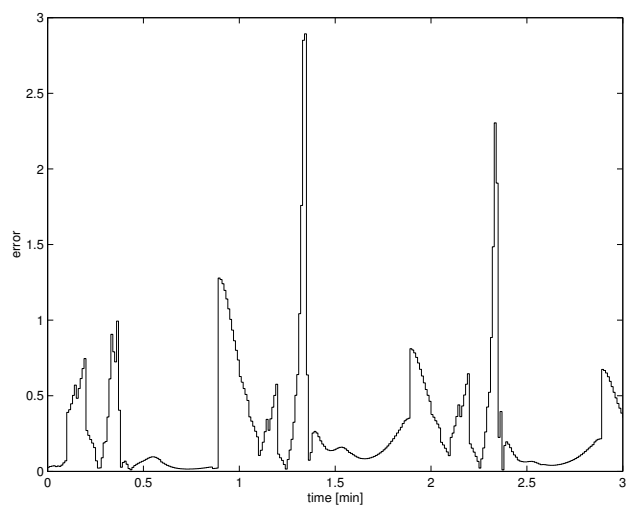

Fig. 5. Phase percentage error $(\bmod 2 \pi)$ estimate.

of the phase time evolution, but still well under $3 \%$ of the full $2 \pi$ available range. Therefore the predicted phase behaviour proves to be quite accurate and its accuracy does not seem to deteriorate as time passes. This results assure us that indeed the phase of the proposed example behaves accordingly to equation (10), when not coupled, and to equation (14), when coupled. As a second step into the analysis of this example an experimental set of thirty oscillators was tested,with the same value of $h=0.01$ [min], at first uncoupled. The $\beta(i)$ distribution was taken as a uniform probability distribution in $\left[0.36\left[\mu \mathrm{Mmin}^{-1}\right], 0.41\left[\mu \mathrm{Mmin}^{-1}\right]\right]$. Without entering in much details, this proposed uniform probability distribution translates into a uniform probability distribution for $\omega(i)$ in $[5.934[\mathrm{rad} / \mathrm{min}], 7.2564[\mathrm{rad} / \mathrm{min}]]$. The standard random number function provided by Matlab executed the selection for each oscillator. The reason why the complete range of $\beta$ value, for which this system example shows oscillatory behaviour, has not been selected is because phase lock behaviour, when coupled, is pursued. The phase dynamics for the uncoupled oscillators

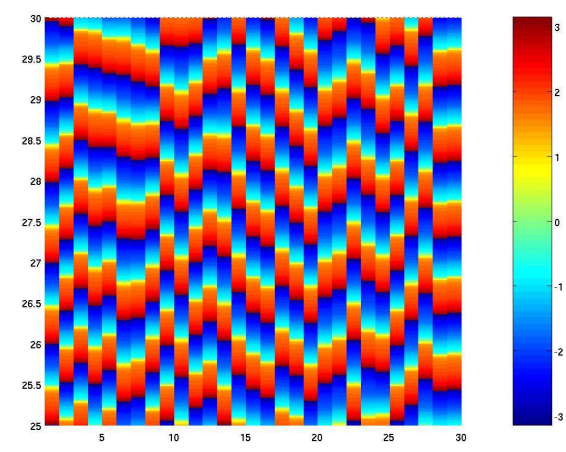

Fig. 6. Uncoupled phase behaviour.

are presented in Figure 6. The horizontal axes correspond to the relative position of each oscillator meanwhile the vertical axes indicates time. The original simulation time was 30 [min], but for clarity only the last $5[\mathrm{~min}]$ are presented in Figure 6 . A colour scale indicates the value of the phase for each individual oscillator, position and time (between $-\pi$ and $\pi$ ). This form of representation allow us to observe at a glance that there is no degree of coordination between them when uncoupled. Afterward the same set of thirty oscillators was coupled together defining, this time, the coupling function in Theorem (1) as:

$$
I_{i}(t)=\frac{Z_{i+1}-Z_{i+1}^{e q}}{\left\|v_{i+1}-v_{i+1}^{e q}\right\|}-\frac{Z_{i}-Z_{i}^{e q}}{\left\|v_{i}-v_{i}^{e q}\right\|}+\frac{Z_{i-1}-Z_{i-1}^{e q}}{\left\|v_{i-1}-v_{i-1}^{e q}\right\|}-\frac{Z_{i}-Z_{i}^{e q}}{\left\|v_{i}-v_{i}^{e q}\right\|}
$$

Where $\left\|v_{i}-v_{i}^{e q}\right\|=\sqrt{\left(Z_{i}-Z_{i}^{e q}\right)^{2}+\left(Y_{i}-Y_{i}^{e q}\right)^{2}}$ and $v_{i}^{e q}=\left(Z_{i}^{e q}, Y_{i}^{e q}\right)$, is the equilibrium point for the $i$-cell. The oscillators located at position 1 and 30 were inter-coupled (forming a one dimensional ring lattice). The results for the global phase behaviour solving the set of 30 coupled ODEs systems is presented, with similar axis definitions as before, in Figure 7. Phase lock behaviour is evident with the onset of a periodic travelling way as colours suggest. On the other hand, to test the result obtained in Theorem (1), $\Delta \tilde{g}$ was obtained for each oscillator with an height $H=0.1$ for each one of them. The resulting function for the first oscillator is presented in the lower panel of Figure (9). To avoid lengthy calculations and also to avoid being forced to work with more than one $\Delta \tilde{g}$ function for each oscillator, linearity was presumed, therefore converting the general $\Delta \tilde{g}$ term into an expression as below:

$$
\Delta \tilde{g}\left[\phi_{i}[k], \omega_{i}, I_{i}[k]\right]=\frac{I_{i}[k]}{0.1} \Delta \tilde{g}\left[\phi_{i}[k], \omega_{i}, 0.1\right]
$$

The resulting predicted global phase behaviour can be seen in Figure (8) and it is strikingly similar to the one obtained solving 


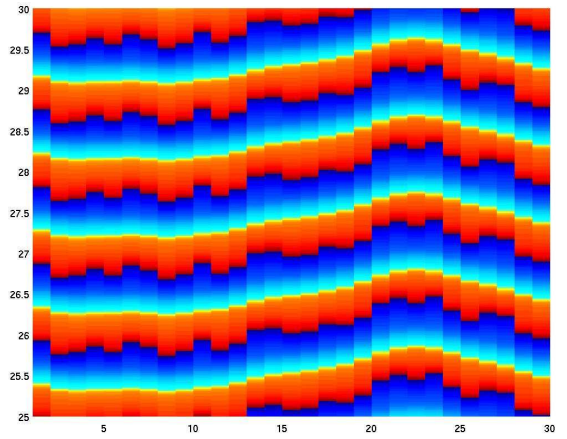

Fig. 7. Coupled phase behaviour described by solving the set of ODE as in equation (11).

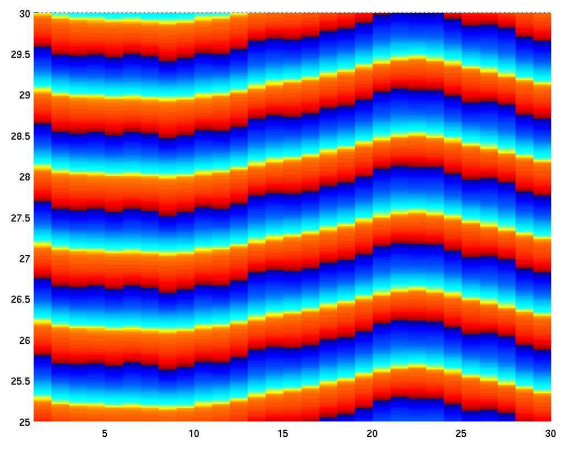

Fig. 8. Coupled phase behaviour obtained through equation (12).
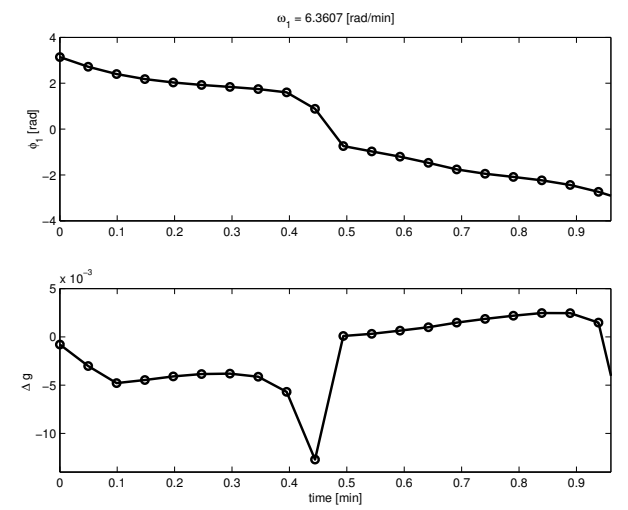

Fig. 9. Data table for the first oscillator, in the upper panel $\phi_{1}$, in the lower panel $\Delta \tilde{g}$ associated to an height of 0.1 in $I_{1}$.

the full set of ODEs equations. This is also remarkable in that confirms in this example, linearity for the coupling term. Finally another point addressed by Theorem (1) is that it allows us to recognise directly what is the superposed effect of the coupling over the independent dynamic of each oscillator.

\section{DisCUSSION AND CONCLUSION.}

In this article a phase model has been inferred from an original state space based model. This alternative model is to be useful when entrainment and synchronism phenomenons arise due to a coupling function. The effect of this coupling function can be observed independently in the proposed global model from the term that arise without coupling. This can be used to study the nature of the coupling between a family of oscillators. Also can be useful in estimate the importance of any such coupling in the behaviour of each separate oscillator when synchronism or more complex interdynamics are present (e.g. quasiperiodic motions).

Future work will include oscillator systems within state spaces of dimension greater than two, alternative definitions for a representative vector-like phase representation and a multivariable version of Theorem (1).

\section{APPENDIX.}

Constant values for the model parameters described in equations (20) and (21):

$$
\begin{array}{ccc}
\nu_{o}=3.4\left[\mu \mathrm{Mmin}^{-1}\right] & \nu_{1}=3.4\left[\mu \mathrm{Mmin}^{-1}\right] & V_{M 2}=50\left[\mu \mathrm{Mmin}^{-1}\right] \\
V_{M 3}=650\left[\mu \mathrm{Mmin}^{-1}\right] & n=2 & m=2 \\
w=4 & K_{2}=1[\mu \mathrm{M}] & K_{r}=2[\mu M] \\
K_{a}=0.9[\mu \mathrm{M}] & k_{f}=1\left[\mathrm{~min}^{-1}\right] & k=10\left[\mathrm{~min}^{-1}\right]
\end{array}
$$

\section{REFERENCES}

[1] S. Banerjee and G, Verghese, Nonlinear Phenomena in Power Electronics: Bifurcations, Chaos, Control and Applications, Wiley-IEEE Press, 2001.

[2] J. D. Crawford and K. T. R. Davies, Synchronisation of globally coupled phase oscillators: singularities and scaling for general couplings, Physica D, Vol. 125, pp. 1-46, 1999

[3] E. Brown, P. Holmes, J. Moehlis, Globally Coupled Oscillator Networks, In: Perspectives and Problems in Nonlinear Science: A Celebratory Volume in Honor of L. Sirovich, E. Kaplan, J. Marsden, K. Sreenivasan, pp. 183-215, Springer Verlag. New York, 2003.

[4] E. Brown, P. Holmes and J. Moehlis, On the Phase Reduction and Response Dynamics of Neural Oscillator Populations, Neural Computation, Vol.16, pp. 673-715, 2004.

[5] G. Dupont, and A. Goldbeter, One-pool model for Calcium oscillations involving Calcium and inositol 1,4,5trisphosphate as coagonists for Calcium release, Cell Calcium, Vol. 14, pp. 311-322, 1993.

[6] J. Guckenheimer, and P. Holmes, Nonlinear Oscillations, Dynamical Systems, and Bifurcations of Vector Field, Springer-Verlag, New York, 1983.

[7] G. Iooss, and D.D. Joseph, Elementary Stability and Bifurcation Theory (second edition), Springer-Verlag, New York, 1990.

[8] E.M. Izhikevich, Weakly Pulse-Coupled Oscillators, FM Interactions, Synchronization, and Oscillatory Associative Memory, IEEE Transactions on Neural Networks, Vol. 10, No. 3, pp. 508-526, May 1999.

[9] E.W. Justh, Control of Large Actuator Arrays using Pattern-Forming systems, Center for Dynamics and Control of Smart Structures, University of Mariland, Ph.D. Thesis report.

[10] H.K. Khalil, Nonlinear Systems (second edition), Prentice Hall, Upper Saddle River, New Jersey, 1996.

[11] Y. Kuramoto, Chemical Oscillations, Waves, and Turbulence, Springer-Verlag, New York, 1984.

[12] S. H. Strogatz, From Kuramoto to Crawford: exploring the onset of synchronization in populations of coupled oscillators, Physica D, Vol. 143, pp. 1-20, 2000

[13] J. Keener and J. Sneyd, Mathematical Physiology, SpringerVerlag,New York, 1998.

[14] A.T. Winfree, The Geometry of Biological Time, Springer Verlag, New York, 1980 . 